\title{
Written information on bipolar affective disorder: the patients' perspective
}

\author{
Daniel C. White, ${ }^{1}$ Sofia Laureano Schelten, ${ }^{1}$ Brendan D. Kelly, ${ }^{1}$ Patricia Casey ${ }^{1}$
}

The Psychiatrist (2010), 34, 418-422, doi: 10.1192/pb.bp.109.027177

${ }^{1}$ Mater Misericordiae University Hospital, Dublin, Ireland

Correspondence to Patricia Casey (profcasey@esatclear.ie)

\begin{abstract}
Aims and method The mental health information available to people with bipolar affective disorder is variable in quality. We conducted a qualitative survey in an urban out-patient department to elicit the opinions of people with bipolar affective disorder on the written information provided by three health information providers.
\end{abstract}

Results Participants' responses were generally positive for each leaflet. The two leaflets rated highest by participants used quality assurance tools. However, 20-30\% had difficulty understanding the leaflets. Medical jargon and verbosity were common criticisms.

Clinical implications Professional bodies such as the Royal College of Psychiatrists play an important role in providing patients with written information. Quality assurance standards should be used in the production of patient information. A tool measuring individuals' appraisal of information may ensure information is appropriate to their requirements.

Declaration of interest A grant was provided by Janssen-Cilag to reimburse individuals for their participation in the study. P.C. is editor of The Psychiatrist.
Providing patients with coherent, relevant information has implications for several aspects of mental healthcare, including engagement with services, concordance with treatment and informed consent. Health information enables patients to communicate more effectively with medical professionals, enabling them to take an active role in making decisions about their treatment, as is recommended by the Royal College of Psychiatrists (www.rcpsych.ac.uk). The growth in mental health information providers may be viewed as a positive development in educating both people with mental illness and other members of the general public. In recent years, the establishment of organisations such as DISCERN (www.discern.org.uk) have published rating instruments to evaluate the quality of information provided to the public, and guidelines to assist information providers in developing relevant, high-quality and evidencebased information for users. Despite the general agreement of health organisations' regarding the benefits of providing patients with written information, there is a paucity of research on their opinions about the available mental health information and this may reflect the absence of user involvement until recently.

Mental health information produced by health organisations for patients varies in its focus and may not be appropriate to its target population. Some qualitative studies have found that certain patient groups wish to have more information regarding the aetiology, treatment options and potential medical complications available to them than is currently available. ${ }^{1}$ Other studies have shown that patient information leaflets must be tested on the target audience to ensure that they are patient oriented, ${ }^{2}$ a finding strongly supported by a study that found a large gap (as much as 5 years) between patient reading levels and the comprehension levels demanded by written materials provided for them. ${ }^{3}$

A randomised controlled trial conducted in an orthopaedic centre in London showed that individuals given information leaflets prior to surgery scored higher on a questionnaire of recall than those who had given verbal consent only. ${ }^{4}$ This has significant implications for the issue of informed consent. There is also evidence that providing people with information about their condition reduces stress. ${ }^{5}$ Among individuals with psychiatric illness there is evidence that, contrary to fears that providing negative information would reduce treatment adherence, the opposite occurred. ${ }^{6}$ The purpose of the present study was to examine patient preferences regarding a selection of the informational literature available to them on bipolar affective disorder and to elucidate what changes would make them more acceptable to the readership.

\section{Method}

The study was conducted in the Mater Misericordiae University Hospital, Dublin, Ireland. Psychiatric out-patients with a diagnosis of bipolar affective disorder who were currently attending out-patient clinics in the hospital or were attending a community clinic were included in the study. 
Individuals were excluded if they were in-patients at the time of the study and if they were currently judged as too unwell to participate in the study. Given that the aim of this study was to examine their preferences for written information, individuals with English or literacy problems were also excluded. The authors acknowledge that literacy difficulties are commonplace in Ireland, affecting approximately $25 \%$ of the adult population according to the National Adult Literacy Association. Further research investigating issues of literacy and patient information in this group is warranted but lies outside the scope of this paper.

All individuals eligible for inclusion were asked to participate in the survey. Participants were contacted either during attendance at the out-patient clinics, by telephone or in writing. Those who agreed to participate in the study were asked to read each information leaflet and complete the questionnaires (Table 1) on each of the three leaflets and to state which they rated as best overall. The leaflets examined were those provided by the Royal College of Psychiatrists (London), a voluntary organisation in Ireland (Aware; www.aware.ie) and a UK-based patient information website run by general practitioners (www.patient.co.uk). Basic demographic data were collected from each participant (age and gender). It took approximately $1 \mathrm{~h}$ to read the leaflets and complete the questionnaire, and participants were advised that they would be reimbursed with a gift voucher to the value of $€ 20$ for their time. Participants were provided with a stamped addressed envelope to return the questionnaires, which were completed at home.

As there was no tool in existence to assess the suitability of information leaflets for patients and their satisfaction with this information, the authors of this study developed a questionnaire for this purpose. Each questionnaire (Table 1) contained 15 statements examining three aspects of the leaflet: comprehensibility; information content; impact of the leaflet on the patient's understanding of their illness. Participants indicated whether they agreed or disagreed with each statement in the questionnaire. The participants were masked to the source of each leaflet. The leaflets and questionnaires were stapled together in a different order and participants were asked to read the leaflets in the order received, so as to reduce order bias. The participants were informed that they could contact D.C.W. with any questions regarding the study.

\section{Results}

A total of 45 people with bipolar disorder were invited to participate in the study, and 27 did so. Of those who participated, 59.3\% ( $n=16)$ were male, and the median age of all participants was 44 years (range 17-70). Mean duration of illness was 16.04 years (s.d. $=9.39$, range 2-40). Those who participated $(n=27)$ did not differ from those who did not participate $(n=18)$ in terms of gender $\left(\chi^{2}=0.805, P=0.527\right)$ or age (Mann-Whitney $U=161.500$, $P=0.059)$. Table 1 displays the number of participants who agreed or disagreed with each statement in the questionnaire (also expressed as a percentage of total responses for each statement). In instances where participants did not make a response for a particular statement, the number of individuals agreeing or disagreeing with the statement was recorded as a proportion of the total number of responses.

No relationship was found between rating 'Which leaflet was best' and gender $\left(\chi^{2}=2.253\right.$, d.f. $\left.=2, P=0.324\right)$, age group $\left(\chi^{2}=5.205\right.$, d.f. $\left.=6, P=0.518\right)$ or the order in which the leaflets were read $\left(\chi^{2}=7.464\right.$, d.f. $\left.=10, P=0.681\right)$. Mean duration of illness was not related to which leaflet each participant rated as best $(F=0.989, P=0.390)$

\section{Which leaflet was best?}

Of the 27 people who participated in the study, 25 (92.6\%) responded to this section of the survey. In total, $52 \%(n=13)$ of the sample rated the leaflet from the Royal College of Psychiatrists as best, with $28 \%(n=7)$ of the sample preferring the leaflet provided by patient.co.uk and the remaining $20 \%(n=5)$ preferring the information leaflet provided on the Aware website.

\section{Comprehensibility}

The majority of respondents found each of the leaflets comprehensible (Table 1); it is noteworthy, however, that $23.1 \%$ of participants felt there were 'more than a few areas' that they could not understand in the patient.co.uk leaflet and almost 30\% thought the Aware leaflet could have been clearer.

\section{Information content}

Respondents were less satisfied with the amount of information contained in the Aware leaflet (80\% satisfied) than the patient.co.uk and Royal College of Psychiatrists leaflets (92.6\% and $92.3 \%$ respectively). Only $53.8 \%$ of respondents felt that the Aware leaflet provided enough information about lithium monitoring and when to contact their doctor, compared with $84.6 \%$ and $92.3 \%$ for the patient.co.uk and the Royal College of Psychiatrists' leaflets respectively. Between 70 and $80 \%$ of participants wanted more information regarding rare but potentially dangerous complications of the medication they were taking for each of the leaflets. The Royal College of Psychiatrists' leaflet performed particularly well in providing information about lifestyle, details of support groups and other sources of information compared with the other two leaflets.

\section{Impact}

Respondents felt that the information contained in the Royal College of Psychiatrists' leaflet would help them to manage their condition more effectively (96.2\% agreed) compared with the Aware and patient.co.uk leaflets (88.9\% and $81.5 \%$ respectively). Between $65 \%$ (Aware leaflet) and $73 \%$ (patient.co.uk leaflet) of participants felt less anxious about having bipolar affective disorder as a result of reading the leaflets. Despite rating the Royal College of Psychiatrists' leaflet as the best overall, respondents felt that the Aware leaflet would be more useful for people recently diagnosed with bipolar affective disorder.

\section{Effects of comprehensibility and information content on impact of leaflets}

The effects of 'comprehensibility' and 'information content' on the 'impact' of the leaflets were explored. The odds ratio (OR) was calculated for each leaflet for the general 
Table 1 Results from questionnaire

\begin{tabular}{|c|c|c|c|}
\hline \multirow[b]{2}{*}{ Area assessed } & \multicolumn{3}{|c|}{ Response to statement, $n / N(\%)$} \\
\hline & Aware & patient.co.uk & $\begin{array}{l}\text { Royal College } \\
\text { of Psychiatrists }\end{array}$ \\
\hline \multicolumn{4}{|l|}{ Comprehensibility } \\
\hline I could understand most of what was mentioned in the leaflet (agree) & $27 / 27(100)$ & $26 / 27(96.3)$ & $26 / 27(96.3)$ \\
\hline There were more than a few areas that I could not understand (disagree) & $20 / 25(80)$ & $20 / 26(76.9)$ & $21 / 25(84)$ \\
\hline The leaflet could have been clearer in what it was saying (disagree) & $17 / 24(70.8)$ & $20 / 25(80)$ & $19 / 24(79.2)$ \\
\hline \multicolumn{4}{|l|}{ Information content } \\
\hline I felt that the information in the leaflet was relevant to me (agree) & $22 / 27(81.5)$ & $25 / 27(92.6)$ & 24/27 (88.9) \\
\hline I was satisfied with the level of information in the leaflet (agree) & $20 / 25(80)$ & $25 / 27(92.6)$ & $24 / 26(92.3)$ \\
\hline I would want to know more about any rare but potentially dangerous & & & \\
\hline complications of my medication (agree) & $21 / 26(80.8)$ & $21 / 27(77.8)$ & $18 / 26(69.2)$ \\
\hline $\begin{array}{l}\text { The leaflet tells me about the need for blood tests, side-effects of } \\
\text { medications, when to contact my doctor (agree) }\end{array}$ & $14 / 26(53.8)$ & 22/26 (84.6) & $24 / 26$ (92.3) \\
\hline The leaflet contained a number of different treatments for my condition, & & & \\
\hline e.g. different medications, psychotherapy (agree) & $20 / 26(76.9)$ & $22 / 25(88)$ & $21 / 25(84)$ \\
\hline The leaflet contained information about lifestyle to help me stay well, & & ) (00) ברוברי & \\
\hline $\begin{array}{l}\text { e.g. avoiding alcohol and drugs (agree) } \\
\text { The leaflet recommended other resources (books/organisations/websites) }\end{array}$ & $21 / 26(80.8)$ & $22 / 25(88)$ & $25 / 26(96.2)$ \\
\hline if I needed to get more information & $26 / 27(96.3)$ & $22 / 26(84.6)$ & $25 / 25(100)$ \\
\hline The leaflet had information on support groups that I could contact (agree) & $25 / 26(96.2)$ & $22 / 25(88)$ & $25 / 25(100)$ \\
\hline \multicolumn{4}{|l|}{ Impact } \\
\hline $\begin{array}{l}\text { I think that this leaflet has given me information that will help me } \\
\text { to manage my condition more effectively (agree) }\end{array}$ & $24 / 27(88.9)$ & $22 / 27(81.5)$ & $25 / 26(96.2)$ \\
\hline Reading the leaflet made me less anxious about my illness (agree) & $17 / 26(65.4)$ & $19 / 26(73.1)$ & $18 / 25(72)$ \\
\hline I would know how to get more information about my condition (agree) & $25 / 27(92.6)$ & $26 / 27(96.3)$ & $26 / 26(100)$ \\
\hline $\begin{array}{l}\text { I think this leaflet would be useful to other people who had recently } \\
\text { been diagnosed with bipolar affective disorder (agree) }\end{array}$ & $25 / 26(96.2)$ & $24 / 27(88.9)$ & $20 / 24(83.3)$ \\
\hline
\end{tabular}

statement measuring the 'impact' of the leaflets 'I think that this leaflet has given me information that will help me to manage my condition more effectively' and each of the statements 'There were more than a few areas that I could not understand' (comprehensibility) and 'I was satisfied with the level of information in the leaflet' (information content). No significant relationship was found between the statements assessing comprehensibility and impact (OR=0.444, 95\% CI 0.032-6.188) for the Aware leaflet. However, a significant relationship was found between information content and impact (OR $=4.889$, 95\% CI $1.304-$ 18.327), with those who were satisfied with the information content, rating the impact of the leaflet higher. No relationship was found between comprehensibility and impact for the patient.co.uk leaflet $(\mathrm{OR}=0.353,95 \%$ CI $0.043-2.867)$ or between information content and impact $(\mathrm{OR}=5.250$, 95\% CI 0.269-102.424). No relationship was found between comprehensibility and impact for the Royal College of Psychiatrists' leaflet $(\mathrm{OR}=1.211$, 95\% CI 1.0001.460), or information content and impact (OR $=1.043,95 \%$ CI 0.960-1.134).

\section{Results from free comments section}

At the end of each questionnaire, participants were invited to make comments. It was envisaged that the statements from the questionnaires would act as prompts to assist participants in completing this part of the study, providing the opportunity to elaborate on their answers or to raise issues felt to be of importance. The comments were categorised into four themes; general, comprehensibility, information content and impact. The general comments category was included to present the overall perceptions of the leaflet, e.g. 'the leaflet was written in a considerate manner' or 'the leaflet was optimistic'. Thirteen participants (48\%) made comments in this section of the questionnaire for the Aware leaflet, 13 people (48\%) for the Royal College of Psychiatrists leaflet and 10 people (37\%) for the patient.co.uk leaflet.

\section{General comments}

Participants responded positively to the three leaflets, particularly the leaflet provided by the Royal College of Psychiatrists.

\section{Comprehensibility}

The Aware leaflet was considered to be verbose by $23.1 \%$ of participants. Although $50 \%$ of respondents commented that the patient.co.uk leaflet was easy to understand, a further $20 \%$ commented that it contained confusing medical jargon, citing as examples 'transcranial magnetic stimulation' and 'vagal nerve stimulation'. A total of $30.8 \%$ of respondents commented that the Royal College of Psychiatrists leaflet was 'clear' or 'easy to understand' and $7.7 \%$ of the participants commented that 'the short paragraphs' made this leaflet 'easier to read', 'especially for people who had difficulty concentrating'.

\section{Information content}

A total of $23.1 \%$ of respondents commented that they found the vignettes in the Aware leaflet useful. However, $23.1 \%$ also commented that the Aware leaflet did not contain enough information about medications and their sideeffects, or information on other support groups or information sources for bipolar affective disorder, whereas 
$23.1 \%$ of participants commented that medications and sideeffects were explained particularly well in the Royal College of Psychiatrists' leaflet. Although $15.4 \%$ of respondents commented that the Royal College of Psychiatrists' leaflet contained a sizeable number of self-help groups, support groups and contacts for further information, $20 \%$ commented that the patient.co.uk leaflet did not contain enough information about support or self-help groups. Thirty per cent of respondents commented that the patient.co.uk leaflet explained medication in a clear and informative manner and $20 \%$ of respondents commented that this leaflet included upto-date information. Also, $30.8 \%$ of respondents commented that the Royal College of Psychiatrists' leaflet contained useful information for family, friends or carers.

\section{Impact}

Referring to the patient.co.uk leaflet, $30 \%$ of respondents commented that they did not like the use of words such as 'antipsychotic' and 'anticonvulsant' medication, and $20 \%$ of respondents commented that they felt anxious being told that the mechanism of action of medications such as lithium and the anticonvulsants were unknown.

\section{Discussion}

This study suggests that the overall quality of information on bipolar affective disorder available in printed form from the selected online health services (Aware, patient.co.uk and the Royal College of Psychiatrists) is of a high standard and is understood by the majority of patients. However, a significant proportion of the study sample, approximately $20-30 \%$, had some difficulty in understanding a number of areas within each leaflet. The participants' comments indicate that concise explanations and the absence of medical jargon may assist their comprehension of written information. This would also help to address the issue of varying literacy levels within patient groups.

Some information that medical professionals regard as desirable and which patients also wish to have, for example, the need for blood tests, side-effects of medication, and when people should seek medical advice is less consistently included in the leaflets of different organisations. The information leaflet provided by the Royal College of Psychiatrists was consistently rated more highly than the other two on its content, suggesting that professional bodies have an important role in patient education.

Of note, the two leaflets that were rated most highly both used quality assurance guidelines produced by internet-based health information organisations; DISCERN and HONcode (Health on the Net Foundation; www.hon.ch/) . With the growing number of internet-based organisations producing health information it is important that doctors take an active role in directing people to websites that use quality assurance instruments in their development. ${ }^{7}$ Websites and organisations usually stipulate if they have used quality assurance instruments in the production of health information leaflets.

A number of issues relating to informed consent were raised in this study. People with bipolar affective disorder wish to know more about the potential side-effects of the medication that they are receiving, an issue of particular relevance if patients are to be involved in decision-making about their treatment. In light of the concerns expressed about specific aspects of medication, such as their mechanism of action, providing clear explanations and information early in the course of the illness may be reassuring and may help to reduce the stigma associated with taking medication, as is evidenced by the participants' dislike of words such as 'antipsychotics' and 'anticonvulsants'. This might also improve treatment adherence rates and ultimately long-term outcomes, as has been demonstrated in research conducted by other medical specialties. ${ }^{8}$

The finding of a significant relationship between information content and impact for the Aware leaflet suggests that special attention should be paid to this when developing leaflets. The wide confidence intervals undermine the robustness of this particular finding, coupled with the failure to identify such a relationship in the other two leaflets, although it was consistent with the findings in another study. ${ }^{9}$ An interesting finding of this study was that although participants rated the Royal College of Psychiatrists' leaflet as the best of the three leaflets, they rated the Aware leaflet highest in terms of its usefulness to people recently diagnosed with bipolar affective disorder. This finding indicates that people's requirements for information may vary depending on their stage of illness.

The absence of a tool to measure patient satisfaction and to gather feedback on the informational sources on bipolar affective disorder necessitated the authors' production of such a tool for the purpose of this study. Although the tool used in this study exhibits face-validity, it may also be appropriate for the development of a tool which demonstrates content validity and internal consistency.

This study has a number of strengths and weaknesses that need to be highlighted. One of its strengths is that its findings can be generalised to patient groups from other inner-city out-patient departments, although the corollary that the needs of patients from other locations such as those from rural areas or those of other socioeconomic groups may not be identified in this study. Participants were masked to the source of the leaflets and there did not appear to be any relationship between the order in which participants read the leaflets and their rating of the leaflets. The main limitation is the study's small size; $60 \%$ of all those with bipolar affective disorder within the study population participated in the survey, however participants and non-participants did not differ on basic demographic data. Nonetheless, the results and statistical analysis in this paper should be interpreted within the context of the sample size. Other limitations include the restriction to one service and the fact that duration of illness was not controlled for in this study. Potential confounding factors such as prior knowledge of bipolar affective disorder, educational and socioeconomic status were also not controlled for. Additionally, as people with English and literacy difficulties were excluded from the study, the results may be biased towards the preferences of individuals with higher educational levels or socioeconomic status. The use of a new instrument to measure views on the leaflets might be considered a weakness. However, since there was no existing instrument, a pragmatic decision was made to use this one, which has face validity. This instrument should be the subject of detailed psychometric examination since it 
could have a place in future studies of this type. The use of Likert scales may have provided more information with respect to the degree of participant agreement or disagreement for each statement; however, the decision to use the agree/disagree scale was used in the interest of simplicity and to avoid central tendency, social desirability and acquiescence biases. Finally, patient involvement in developing the questionnaire would have provided further assurance that the study elicited individuals' preferences for information regarding their illness and this step should be considered in the development of any such tool.

In summary, this study emphasises the important role of mental health professionals in directing patients towards written information and internet websites. Furthermore, professional mental health organisations should be involved in developing or revamping such informational tools. There is a strong argument to be made for involving patients in such initiatives in the future.

\footnotetext{
About the authors

Daniel C. White, Registrar, Mater Misericordiae University Hospital, and Special Lecturer, University College Dublin, Sofia Laureano Schelten, Research Psychologist, Mater Misericordiae University Hospital, Brendan D. Kelly, Consultant Psychiatrist, Mater Misericordiae University Hospital, and Special Lecturer, University College Dublin, Patricia Casey, Mater Misericordiae University Hospital, and Professor of Psychiatry, University College Dublin, Ireland.
}

\section{References}

1 Prinjha S, Chapple A, Herxheimer A, McPherson A. Many people with epilepsy want to know more: a qualitative study. Fam Pract 2005; 22: 435-41.

2 Dickinson D, Raynor DK, Duman M. Patient information for medicines: using consumer testing to determine the most effective design. Patient Educ Couns 2001; 43: 147-59.

3 Davis TC, Crouch MA, Wills G, Miller S, Abdehou DM. The gap between patient reading comprehension and the readability of patient education materials. J Fam Pract 1990; 31: 533-8.

4 Ashraff S, Malawa G, Dolan T, Khanduja V. Prospective randomised controlled trial on the role of patient information leaflets in obtaining informed consent. ANZ J Surg 2006; 76: 139-41.

5 Kitamura T. Stress reductive effects of information disclosure to medica and psychiatric patients. Psychiatry Clin Neurosci 2005; 59: 627-33.

6 Hornung WP, Klingberg S, Feldmann R, Schonauer K, Schulze Mönking $\mathrm{H}$. Collaboration with drug treatment by schizophrenic patients with and without psychoeducational training: results of a 1-year follow-up. Acta Psychiatr Scand 1998; 97: 213-9.

7 Meredith $\mathrm{P}$, Emberton $\mathrm{M}$, Wood $\mathrm{C}$. New directions in information for patients. BMJ 1995; 311: 4-5.

8 Greenfield C, Kaplan S, Ware JE. Expanding patient involvement in care. Effects on patient outcomes. Ann Intern Med 1985; 102: 520-8.

9 Fallowfield LJ, Hall A, Maguire GP, Baum M. Psychological outcomes of different treatment policies in women with early breast cancer outside a clinical trial. BMJ 1990; 301: 575-80.

\title{
Evaluation of antipsychotic information on UK schizophrenia websites
}

\author{
Gazala Akram, ${ }^{1}$ Anne Boyter, ${ }^{2}$ Alison Thomson ${ }^{2}$
}

The Psychiatrist (2010), 34, 422-426, doi: 10.1192/pb.bp.109.026856

${ }^{1}$ NHS Greater Glasgow \& Clyde; ${ }^{2}$ University of Strathclyde, Glasgow Correspondence to Gazala Akram (gazala.akram@strath.ac.uk)

\begin{abstract}
Aims and method To evaluate the quality of information about medication on 33 UK schizophrenia websites using a bespoke tool known as the Strathclyde Website Evaluation Form (SWEF).
\end{abstract}

Results The mean total SWEF score was 26 out of a maximum of 52 . For website content, the mean score was 15.3 out of a maximum of 32 . Most websites mentioned antipsychotics $(n=23)$, but only 10 described the differences between typical and atypical classes. Three websites mentioned the different injectable formulations. Information about side-effects, particularly from atypicals was limited. Comprehensive details about clozapine including the blood monitoring requirements were found on two websites. Currency was reasonable with 20 websites being less than 3 years old.

Clinical implications Information about antipsychotic medication on UK schizophrenia websites is basic and incomplete.

Declaration of interest None.
The internet is increasingly used as a source of healthrelated information. ${ }^{1}$ However, the content and quality of many health-related websites has often been characterised as poor. ${ }^{2}$ For example, one review of 79 studies, $^{3}$ in which 5941 websites were evaluated, found that the quality of the material was 'deemed to be a problem' in $70 \%$ of the 